\title{
Aedes aegypti Pupae are Highly Susceptible to Infection by Metarhizium anisopliae Blastospores
}

\author{
Aline Teixeira Carolino ${ }^{1}$ (D) Simone Azevedo Gomes ${ }^{1}$ D , Thais Bercot Pontes \\ Teodoro $^{1}$ (D), Thalles Cardoso Mattoso ${ }^{2}$ and Richard lan Samuels ${ }^{1 *}$ (iD
}

${ }^{1}$ Department of Entomology and Plant Pathology, State University of North Fluminense. Alberto Lamego Avenue, 2000, Parque California, Campos dos Goytacazes, Rio de Janeiro, Brazil. ${ }^{2}$ Federal University of Roraima, Department of Crop Science. Boa Vista, Roraima, Brazil.

\begin{abstract}
The mosquito Aedes aegypti vectors arboviruses such as urban yellow fever, dengue, chikungunya and Zika. Reducing the vector population is still the most effective method to decrease the spread of arboviruses. Fungi are the main natural disease agents of insects. The present study compared the virulence of conidia and blastospores of the entomopathogenic fungus Metarhizium anisopliae against A. aegypti pupae. The pupae were obtained by collecting eggs using "ovitraps" deployed in an urban environment. M. anisopliae conidia were produced using solid media and blastospores were produced by inoculating conidia in liquid culture. Blastospores were more virulent against pupae than conidia, and no pupae survived twenty-four hours after exposure to this type of propagule. Large quantities of mucilage were produced by the blastospores in the presence of the pupae. Exposure of pupae to conidial suspensions resulted in $57 \%$ survival at $24 \mathrm{~h}$ and $23 \%$ at $48 \mathrm{~h}$. A proportion of the adults, which emerged from pupae exposed to conidia, succumbed to infection. This is the first study to demonstrates the infection of $A$. aegypti pupae by the entomopathogenic fungus $M$. anisopliae, showing that this stage of development can also be targeted by biological control agents.
\end{abstract}

Keywords: Virulence, Fungus, Vector, Biological control, Pupa, Dengue.

\footnotetext{
*Correspondence: richardiansamuels@gmail.com

(Received: 28 May 2019; accepted: 04 July 2019)

Citation: Aline Teixeira Carolino, Simone Azevedo Gomes, Thais Bercot Pontes Teodoro, Thalles Cardoso Mattoso and Richard lan Samuels, Aedes aegypti pupae are highly susceptible to infection by Metarhizium anisopliae blastospores, J Pure Appl Microbiol., 2019; 13(3): 1629-1634. https://doi.org/10.22207/JPAM.13.3.36

C The Author(s) 2019. Open Access. This article is distributed under the terms of the Creative Commons Attribution 4.0 International License which permits unrestricted use, sharing, distribution, and reproduction in any medium, provided you give appropriate credit to the original author(s) and the source, provide a link to the Creative Commons license, and indicate if changes were made.
} 


\section{INTRODUCTION}

The arboviruses urban yellow fever, dengue, Zika and chikungunya, vectored by the mosquito Aedes aegypti, continue to present serious public health concerns worldwide ${ }^{1,2}$. For decades synthetic insecticides have been used to control insect vector populations. However, the use of these chemicals has caused serious environmental impact and negative effects on human health. As a consequence of indiscriminate chemical use, studies indicate that a large percentage of the mosquito population has developed resistance to these insecticides ${ }^{3,4}$. An alternative to chemical agents for mosquito control is the use of microbiological agents in integrated pest management programs. One of the main advantages of using biological products is their selective action, being harmless to human health and to the environment ${ }^{5}$. The use of Bti (Bacillus thuringiensis var. israelensis) based biolarvicides is an effective alternative for the reduction of mosquito populations ${ }^{6}$. However, as these bacteria need to be ingested to kill the target insect, Bti is ineffective for the control of eggs, pupae and the adult stages ${ }^{7,8}$. A more comprehensive option for insect control is the use of entomopathogenic fungi. $M$. anisopliae is highly promising for the control of $A$. aegypti, as this fungus infects eggs, larvae and adults $\mathrm{s}^{9,10,11}$.

The potential to control all stages of the mosquito life cycle is important for efficient integrated pest management, reducing the number of adults that transmit diseases to the human population ${ }^{12}$. To date, no study has investigated the microbial control of the pupal phase of the mosquito life cycle. Here we investigated the pathogenicity and virulence of $M$. anisopliae blastospores and conidia against $A$. aegypti pupae.

\section{METHODS \\ Mosquito maintenance}

A. aegypti eggs were collected in the field using "ovitraps". The ovitraps were made from 700 $\mathrm{mL}$ black plastic plant pots with 4 hardboard strips vertically attached to the pots. These traps were half-filled with tap water and placed in shaded areas around the Campus of the State University of North Fluminense (UENF). After one week, all of ovitraps were collected and the wooden strips analyzed in the laboratory, using a dissecting microscope, to confirm the presence of mosquito eggs. Subsequently, the strips were immersed in trays with water to stimulate the hatching of the larvae. Larvae were maintained on ground fish food until they developed into pupae. These pupae ( 1 day old) were then immediately used in the following experiments.

\section{Preparation of suspension of $M$. anisopliae conidia and blastospores}

For the conduction of the assays, $M$. anisopliae isolate LEF 2000 was used. This isolate was obtained from a soil sample in Campos dos Goytacazes, Brazil. Following purification of this fungus by consecutive reisolation, a monosporic culture was obtained by conventional methods. The fungus was then identified from conidiophore morphology using taxonomic keys. This isolate is currently maintained in the fungal collection of the Insect Pathology Sector at the State University of North Fluminense.

M. anisopliae conidia were obtained from culture medium containing potato, dextrose and agar (PDA) and maintained in an incubator for ten days at $27{ }^{\circ} \mathrm{C}$ and $70 \% \mathrm{RH}$. After growth of the fungus, the conidia were carefully removed from the culture medium with the aid of a spatula and a suspension was prepared using Tween 20 $(0.01 \%)$ at the concentration of $1 \times 10^{7}$ conidia $\mathrm{mL}^{-1}$, estimated using a Neubauer hemocytometer.

Blastospores of $M$. anisopliae were produced in culture medium containing $3 \%$ corn steep liquor (Sigma-Aldrich, Brazil), $4 \%$ yeast extract and $4 \%$ glucose. The liquid medium (50 $\mathrm{mL}$ ) was placed in Erlenmeyer flasks $(250 \mathrm{~mL})$. A volume of $500 \mu \mathrm{L}$ of the conidial suspension ( $\left.1 \times 10^{7} \mathrm{~mL}^{-1}\right)$ was added to the liquid medium and incubated at $27^{\circ} \mathrm{C}$ in orbital shaker at $152 \mathrm{rpm}$. After three days, the blastospores were separated from the hyphal fragments present in the culture using a "Miracloth" filter (Sigma-Aldrich, Brazil). The concentration of blastospores in the suspension was also estimated using Neubauer hemocytometer. A final concentration containing $1 \times 10^{7}$ blastospores $\mathrm{mL}^{-1}$ was used in the assays.

\section{Infection bioassays of $A$. aegypti pupae}

After larvae hatched, the rearing was monitored daily for selection of newly formed pupae. The pupae were placed in 4 plastic containers $(200 \mathrm{~mL})$, each with 10 individuals 
( $N=40$ pupae per treatment). A volume of 50 $\mathrm{mL}$ of the conidial or blastospore suspension was added to the vessel. Controls was carried out with water only (blastospore treatments) or Tween only (conidial treatments). The treatments were maintained on a laboratory bench at a temperature of approximately $27{ }^{\circ} \mathrm{C}$. Pupal survival was monitored for a period of 3 days, the maximum time observed to normally complete this stage of the life cycle under laboratory conditions. All assays were carried three times.

In an additional assay, 40 pupae that had been treated with conidia, as stated above, were used to evaluate the survival rate of adults emerging after pupal infection. The survival rates of these newly emerged adults were compared with the control group, which had only been exposed to Tween 20 (0.01\%).

\section{Statistical analysis}

All experiments were carried out using 40 insects per treatment or control group, with all experiments carried out three times. Differences in survival rates between groups and the median survival time $\left(\mathrm{S}_{50}\right)$ were calculated and analyzed using ANOVA and Kaplan-Meier analysis. The significance was determined using Log-rank pair-wise comparisons at the $95 \%$ significance level. Insects which survived the 3 days of the experiment was regarded as "censored".

\section{RESULTS}

The result of this study demonstrated for the first time the infection of pupae by blastospores and conidia of the entomo-pathogenic fungus M. anisopliae. Significant differences between treatments were observed using the Log-Rank test $\left(X^{2}(3)=432.3, P<0.0001\right)$. Twenty-four hours after exposing pupae to blastospore suspensions, $100 \%$ of the pupae had died (Fig. 1). The presence of mucilage was observed covering the entire pupal integument 24h post-infection (Fig. 2). Blastospores and mucilage covered the entire surface of the pupae (Figure 2A and 2B). Mucilage showed strong adherence since it was not possible to remove from the pupae in the water (Fig. 2A and $2 \mathrm{~B})$. Conidiogenesis was observed on the pupal

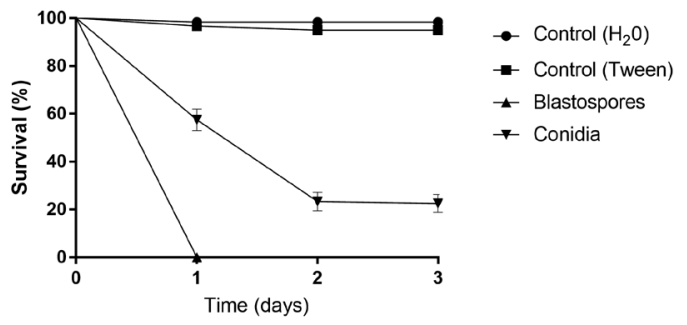

Fig. 1. Survival curves of $A$. aegypti pupae following exposure to blastospore and conidial suspensions $\left(1 \times 10^{7}\right.$ $\mathrm{mL}^{-1}$ ) during 3 days. Controls group were exposed to distilled water or Tween 20 (0.01\%).

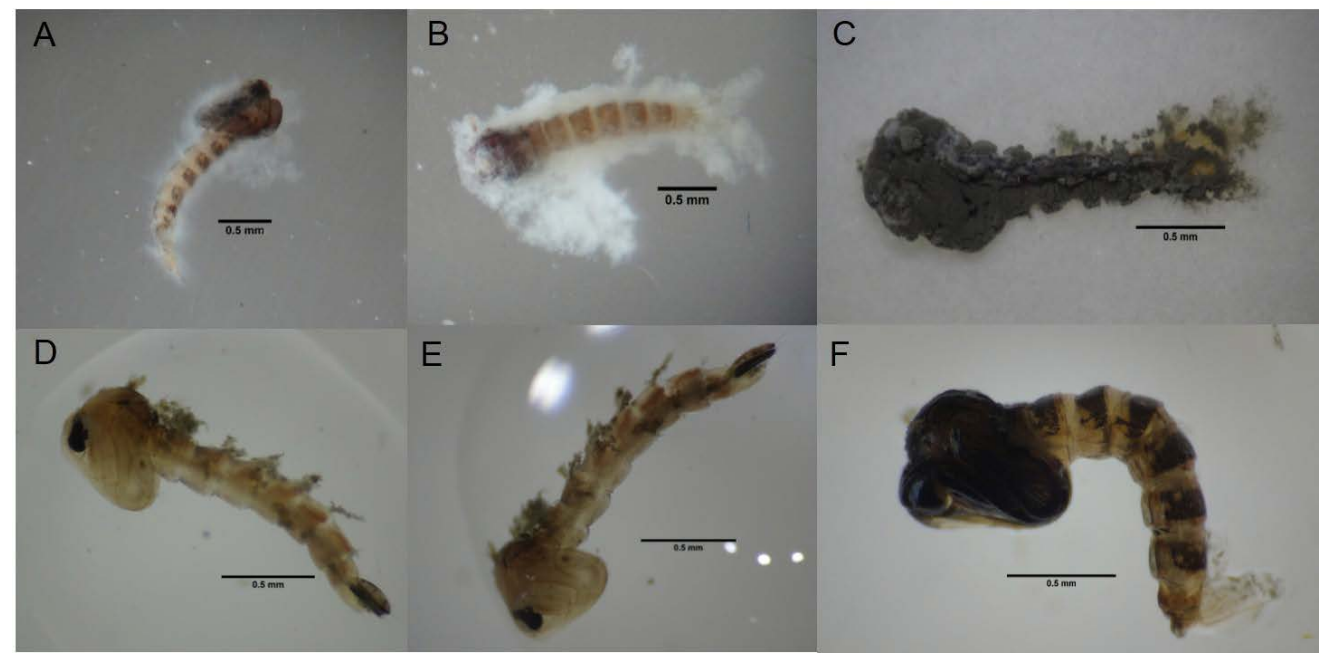

Fig. 2. Blastospores infecting Aedes aegypti pupae with copious amounts of mucilage secreted by the fungus (A and B). After infection by blastospores, conidial sporulation was observed on dead pupae (C). Pupae infected with Metarhizium anisopliae conidia showed no mucilage production and no sporulation was observed on the insect integument (D - F) 
cuticle when blastospores produced conidiophores (Fig. 2C).

In the conidial treatment, 24 hours after exposition to the fungus, pupal survival was $57.5 \%$. After $48 \mathrm{~h}$ it decreased to $23.3 \%\left(\mathrm{~S}_{50}=2\right.$ days $)$. Pupae infected with $M$. anisopliae conidia showed no mucilage and no conidia were observed attached to the surface of the pupae. Also, no sporulation was observed on the insect integument (Fig. 2D F). On the third day of evaluation, it was possible to observe the emergence of a low number of adults from the pupae that had survived this treatment.

Control pupae developed normally, and adult mosquitoes were observed at the final evaluation stage of the experiment. On the third day, $98.3 \%$ and $95 \%$ survival rates were observed in the water and Tween control groups respectively. Analysis of variance showed no difference between the control groups $(P=0.264)$.

In the assay to monitor adult survival, $27.5 \%$ of the pupae that had been exposed to conidia resulted in adult emergence. However, $54.5 \%$ of these adults subsequently died over the next three days and sporulation of fungi could be observed on all of these adults (data not shown here). Over $90 \%$ of pupae in the control treatments were viable with adults emerging normally and surviving for more than 30 days.

\section{DISCUSSION}

The present study showed for the first time that blastospores and conidiospores of the entomopathogenic fungus $M$. anisopliae are virulent to $A$. aegypti pupae. Blastospores were significantly more virulent than conidiospores (conidia) of the same fungal isolate.

After the pupae succumbed to infection by both conidia and blastospores, it could be observed that the pupae had an unusual anatomical appearance (Figure 2), which indicated an acceleration of the molting process to the adult stage, possibly in an attempt to escape the infection. The third-instar nymphs of aphids infected with Lecanicillium attenuatum also showed an accelerated molting process, resulting in reduced infection rates ${ }^{13}$. Insects can modify their behavior as a survival strategy in an attempt to avoid pathogen infection. Triggering successive molting, adjusting the body temperature to inhibit fungal grow or, as in social insects, allogrooming and abandoning the nest, are all infection avoidance strategies ${ }^{13,14,15}$. Anopheles gambiae larvae infected with the bacteria Asaia, also showed an accelerated developmental rate through the four larval stages, reaching the pupal stage almost 48 hours faster than non-infected larvae ${ }^{16}$. The molting process can inhibit the pathogen as phenol oxidase enzymes present in the molting fluid remain active in the newly formed cuticle for an extended period, binding to the components of the fungal cell wall (chitin and glucan), inhibiting the germination of the fungus ${ }^{17}$. Interestingly, blastospores of $M$. brunneum stained with Calcofluor White, a dye widely used to stain fungal cell wall components, showed that blastopsores had little or no glucan ${ }^{18}$. This fact may have resulted in a reduced influence of phenol oxidase enzymes present in newly formed cuticle of the pupae, allowing rapid adhesion and consequent infection. An interesting observation in the present study was that some of the pupae that had apparently "escaped" conidial infections and metamorphosed to adults, died soon after emerging, with subsequent conidiogenesis on the cadaver, demonstrating that the adults had died from fungal infection. This may suggest that although the pupae had successfully molted to the adult stage, the fungus was developing within the hemolymph, resulting in adult infection and death.

In an aquatic environment, the mucilage secreted by $M$. anisopliae blastospores facilitates the adhesion of the fungus to the insect's integument, which accelerates infection and reduces the time to $\mathrm{kill}^{18}$. The secretion of mucilage may result in rapid pupal mortality, compromising respiration by blocking of the respiratory trumpets.

Conidia can also produce a thin layer of mucilage that is secreted by mature appressorium to reinforce the attachment to the insect integument ${ }^{19}$. However, conidia were found to bind poorly to weakly polar surfaces, even after a 24 hour exposure period ${ }^{20}$. This corroborates the results of the present study and those of Greenfield et al. ${ }^{21}$, who reported that adhesion of $M$. anisopliae conidia to aquatic mosquito larvae was low when compared with that on terrestrial insect integument (Tenebrio molitor). 
The infection route of blastospores or conidia when attacking mosquito pupae has not yet been investigated, but the results of the present study indicate a novel mode of action of the blastospores, which have great potential for the control of this stage of the mosquito life cycle, due to rapid mortality of the host. Insect pupae are normally considered to be a difficult target for control, as they do not feed and this phase only lasts approximately 3 days in the case of $A$. aegypti.

The integument of the pupae is more sclerotized than that of the larvae. For biological control agents such as Bti, regularly used to control the larval phase, ingestion is necessary to cause infection ${ }^{6,7}$. Previous studies have shown that conidia of the entomopathogenic fungus $M$. brunneum, when used against $A$. aegypti larvae in an aquatic environment, have low adhesion rates to the integument ${ }^{22}$. However, the presence of conidia in the intestine of $A$. aegypti larvae was observed following ingestion of these propagules and death was attributed to stress caused by proteolytic enzymes released by the conidia ${ }^{22}$.

Efficiently controlling the immature forms of the vector reduces the chances of adult emergence, and thus decreases the incidence of disease transmission. Pupae are highly susceptible to $M$. anisopliae blastospore infection, which makes this propagule a promising tool for $A$. aegypti biological control strategies or for incorporation in an integrated vector management program.

This study shows that blastospores of $M$. anisopliae are efficient in preventing the development of $A$. aegypti pupae, with $100 \%$ pupal mortality in only twenty-four hours. The study shows the possibility of using fungi to control this phase of development of the mosquito. Pupae are normally more resistant to biological control measures due to the short duration of this phase of the life cycle, therefore the infection process needs to be very quick and efficient. By killing the immature forms of this vector, we can reduce the population of the adults, lowering the vectorial capacity of this insect.

\section{ACKNOWLEDGMENTS}

FAPERJ for the post-doctoral fellowship awarded to ATC. UENF for the studentships award to TBPT and the post-doctoral fellowship awarded to TCM. CNPq for the studentship awarded to SAG. RIS is a CNPq research fellow.

\section{CONFLICT OF INTEREST}

The authors declares that there is no conflict of interest.

\section{AUTHORS' CONTRIBUTION}

ATC designed and performed the experiments, analyzed the data and wrote the initial draft of the paper. SAG, TBPT and TCM helped perform the experiments and data analysis. RIS supervised the research and corrected the original draft.

\section{FUNDING}

FAPERJ: E-26/201.345/2016; E-26/203.066/2016; E-26/201.336/2016. CNPq: 431766/2016-9.

\section{DATA AVAILABILITY}

All data is freely available from the corresponding author on request.

\section{ETHICS STATEMENT}

Not applicable

\section{REFERENCES}

1. World Health Organization, Global vector control response 2017-2030. https://www.who.int/vectorcontrol/qa-vector-control/en/, Accessed 21 Dec, 2018.

2. Brazilian Ministry of Health, Monitoramento dos casos de dengue, febre de chikungunya e doenca aguda pelo virus Zika ate a Semana Epidemiológica. http:// portalarquivos 2.saude. gov.br/images/pdf/2018/ agosto/07/2018-040.pdf, Accessed 11 Jan, 2019.

3. Braga I A, Valle D. Aedes aegypti: historico do controle no Brasil. Epidemiologia $e$ Servicos de Saude, 2007; 16 (2): 113-118. https://doi.org/10.5123/S1679-49742007000400007.

4. Wondji C S, Silva W A P P, Hemingway J, Ranson H, Karunaratne S H P P. Characterization of knockdown resistance in DDT and pyrethroid-resistant Culex quinquefasciatus populations from Sri Lanka. Trop. Med. Int. Health, 2008; 13 (4): 548-555. https://doi.org/10.1111/j.1365-3156.2008.02033.x.

5. Bale J S, Van Lenteren J C, Bigler F, Biological control and sustainable food production. Philos Trans Soc, B, 2008; 363: 761-776. https://doi.org/10.1098/rstb.2007.2182.

6. Lacey L A, Grzywacz D, Shapiro-llan D I, Frutos R, Brownbridge M, Goettel M S. Insect pathogens as biological control agents: back to the future. 
J Invertebr Pathol, 2015; 132: 1-41. https://doi.org/10.1016/j.jip.2015.07.009.

7. Lacey LA. Bacillus thuringiensis serovariety israelensis and Bacillus sphaericus for mosquito control. J Am Mosq Control Assoc, 2007; 23 (2): 133-163. https://doi.org/10.2987/8756-971X(2007)23[133:BT SIAB]2.0.CO;2.

8. Pigott C R, Ellar D J. Role of Receptors in Bacillus thuringiensis crystal toxin activity. Microbiol. Mol. Biol. Rev, 2007; 71 (2): 255-281. https://doi.org/10.1128/MMBR.00034-06.

9. LuzC, Tai MHH, SantosAH, Silva H HG. Impact of moisture on survival of Aedes aegypti eggs and ovicidal activity of Metarhizium anisopliae under laboratory conditions. Mem. Inst. Oswaldo Cruz, 2008; 103 (2): 214-215. https://doi.org/10.1590/S0074-02762008000200016.

10. Carolino A T, Paula A R, Silva C P, Butt T M, Samuels R I. Monitoring persistence of the entomopathogenic fungus Metarhizium anisopliae under simulated field conditions with the aim of controlling adult Aedes aegypti (Diptera: Culicidae). Parasit Vectors, 2014; 7 (198): 1-7. https://doi.org/10.1186/1756-3305-7-198.

11. Gomes S A, Paula A R, Ribeiro A, Moraes COP, Santos J W A B, Silva C P, Samuels R I. Neem oil increases the efficiency of the entomopathogenic fungus Metarhizium anisopliae for the control of Aedes aegypti (Diptera: Culicidae) larvae. Parasit Vectors, 2015; 8 (669): 1-8. https://doi.org/10.1186/s13071-015-1280-9.

12. Zara A L S A, Santos S M, Oliveira S F, Carvalho R G, Coelho GE. Estratégias de controle do Aedes aegypti: uma revisão. Epidemiologia e Servicos de Saude, 2016; 25 (2): 391-404.

13. Kim J J, Roberts D W. The relationship between conidial dose, moulting and insect developmental stage on the susceptibility of cotton aphid, Aphis gossypii, to conidia of Lecanicillium attenuatum, an entomopathogenic fungus. Biocontrol Sci Technol, 2012; 22 (3): 319-331. https://doi.org/10.1080/09583157.2012.656580.

14. Jaronski S T, Goettel M S. Development of Beauveria bassiana for control of grasshoppers and locusts. Mem Ent Soc Canada, 1997; 171: 225-231. https://doi.org/10.4039/entm129171225-1.

15. Heinze J, Walter B. Moribund ants leave their nests to die in social isolation. Curr Biol, 2010; 20: 249-252. https://doi.org/10.1016/j.cub.2009.12.031.

16. Mitraka E, Stathopoulos S, Sden-Kiamos I, Christophides G K, Louis C. Asaia accelerates larval development of Anopheles gambiae. Pathog Global Health, 2013; 107 (6): 305-311. https://doi.org/10.1179/2047773213Y.0000000106.

17. Zhang J, Huang W, Yuan C, Lu Y, Yang B, Wang CY, Zhang P, Dobens L, Zou Z, Wang C, Ling E. Prophenoloxidasemediated ex vivo immunity to delay fungal infection after insect ecdysis. Front Immunol, 2017; 8: 1-14. https://doi.org/10.3389/fimmu.2017.01445.

18. Alkhaibarl A M, Carolino A T, Yavasoglu S I, Maffeis T, Mattoso T C, Bull J C, Samuels RI, Butt T M. Metarhizium brunneum blastospore pathogenesis in Aedes aegypti larvae: attack on several fronts accelerates mortality. PLoS Path, 2016; 12 (7): e1005715. https://doi.org/10.1371/journal.ppat.1005715.

19. St Leger R J, Cooper R M, Charnley A K. Production of cuticle-degrading enzymes by the entomopathogen Metarhizium misopliue during infection of cuticles from Calliphora vomitoria and Manduca sexta. J Gen Appl Microbiol, 1987; 133: 1371-1382. https://doi.org/10.1099/00221287-133-5-1371.

20. Holder D J, Keyhani N O. Adhesion of the entomopathogenic fungus Beauveria (Cordyceps) bassiana to substrata. J Appl Environ Microbiol, 2005; 71 (9): 5260-5266. https://doi.org/10.1128/AEM.71.9.5260-5266.2005.

21. Greenfield B P J, Lord A M, Dudley E, Butt TM. Conidia of the insect pathogenic fungus, Metarhizium anisopliae, fail to adhere to mosquito larval cuticle. R. Soc Open Sci, 2014; 1: 140193. https://doi.org/10.1098/rsos.140193.

22. Butt T M, Greenfield B P J, Greig C, Maffeis T G G, Taylor J W D. Metarhizium anisopliae pathogenesis of mosquito larvae: a verdict of accidental death. PloS One, 2013; 8 (12): e81686. https://doi.org/10.1371/journal.pone.0081686. 